\title{
On the relationship between the mercury-manganese stars and the metallic-lined stars
}

\author{
Saul J. Adelman ${ }^{1}$, A. S. Adelman ${ }^{2}$, and O. I. Pintado ${ }^{3, \star}$ \\ 1 Department of Physics, The Citadel, 171 Moultrie Street, Charleston, SC 29409, USA \\ e-mail: adelmans@citadel.edu \\ 2 Department of Biometry and Epidemiology, Medical University of South Carolina, 171 Ashley Avenue, Charleston, \\ SC 29425, USA \\ e-mail: adelmaas@musc.edu \\ 3 Departamento de Fisica, Facultad de Ciencias Exactas y Tecnología, Universidad Nacional de Tucumán, \\ Av. Independencia 1800, 4000 San Miguel de Tucumán, Argentina \\ e-mail: opintado@tucbbs.com.ar \\ Received 23 July 2002 / Accepted 7 October 2002
}

\begin{abstract}
An HR diagram indicating the positions of the HgMn and the Am stars analyzed by the senior author and his collaborators shows that the coolest $\mathrm{HgMn}$ stars and the hottest Am stars are found on the same stellar evolutionary tracks and hence the former must evolve into the later. The explanation of the dividing line between these two types of nonmagnetic chemically peculiar stars where the $\mathrm{Hg}$ abundances suddenly change their degree of overabundance is a major test of the theories which try to explain the anomalous abundances of such stars. Some other important relationships are found which can also serve as tests of theories which purport to explain the properties of these stars.
\end{abstract}

Key words. stars: chemically peculiar - stars: Hertzsprung-Russell (HR) diagram and C-M diagrams - methods: statistical

\section{Introduction}

The non-magnetic Chemically Peculiar stars of the Upper Main Sequence are divided into the two major classes the hotter mercury-manganese ( $\mathrm{HgMn})$ stars and the cooler metalliclined (Am) stars. They are, respectively, peculiar B and peculiar A and early $\mathrm{F}$ main sequence band stars for which magnetic fields are not found in unpolarized light with Zeeman analyzers. Many class members belong to binary systems and rotate more slowly than stars with normal or solar chemical compositions. A few even hotter stars are thought to be the continuation of this sequence. Various investigators (e.g., Adelman \& Albayrak 1998; Wahlgren \& Dolk 1998) have tried to find transitional stars as well as how one sequence merges into another. The boundary between the two types of stars has been known to be close to $T_{\text {eff }}=10000 \mathrm{~K}$ for quite some time (e.g., Adelman et al. 1984). For the HgMn stars linear correlation analyses studies (e.g. in the photographic region, Adelman 1989; Adelman et al. 2001; Pintado \& Adelman 2001 and in the ultraviolet region, Smith \& Dworetsky 1993) found that the

Send offprint requests to: S. J. Adelman,

e-mail: adelmans@citadel.edu

* Member of Carrera del Investigador del Consejo Nacional de Investigaciones Científicas y Técnicas de la República Argentina and Visiting Astronomer at Complejo Astronómico El Leoncito operated under agreement between Consejo Nacional de Investigaciones Científicas y Técnicas de la República Argentina and the National Universities of La Plata, Córdoba and San Juan. abundances of some elements correlate with those of other elements and with effective temperature and surface gravity. The results from these two spectral regions complement and supplement one another. Certain correlations, such as the Mn abundance being a function of $T_{\text {eff }}$, are present in all of the studies, but others depend on which stars are included. On the other hand some abundances do not follow any trends. Many current theories, which purport to explain their abnormal abundances, are based upon radiative diffusion in relatively quiescent atmospheres (see, e.g., Michaud \& Proffitt 1993). Light induced drift is an alternative explanation. As these stars evolve, we would expect that their abnormal abundances should slowly change with time (see, e.g., Hui-Bon-Hoa 2000).

\section{The HR diagram}

In an attempt to find evidence for evolution with age since the Zero Age Main Sequence (ZAMS), we investigated $\mathrm{HgMn}$ and Am stars which were analyzed by the first author and his collaborators. We studied single stars and the primaries of binaries which can be treated as if they are single. We obtained $V$ magnitudes and Strömgren photometry from the SIMBAD database and parallaxes from the Hipparcos satellite (ESA 1997). These stars are more sharp-lined than their class means. We used the effective temperatures of Adelman \& Rayle (2000) and when not available calculated them from the Strömgren photometry using the program of Napiwotzki et al. (1993) and applied corrections from Adelman \& Rayle (2000). 
They are similar to those of the elemental abundance analyses listed in Table 1.

If we assume that the solar Bolometric magnitude is -4.74 following Bessell et al. (1998) and use their Bolometric corrections, then we can find absolute luminosities. Given that the $V$ magnitudes are typically known to $0.01 \mathrm{mag}$, the random errors in the luminosities are due to those of the parallaxes. The effective temperatures are known to an uncertainty of $200 \mathrm{~K}$ or less (Lemke 1989) which corresponds to 0.01 in $\log T_{\text {eff }}$ at $10000 \mathrm{~K}$.

In both luminosity and effective temperature there are potential systematic errors which could produce shifts in the stellar positions in a HR diagram, but the relative stellar positions, even if these errors are present, will be changed by much smaller amounts. The Bolometric correction for the Sun is still a source of some controversy. The effective temperatures of these stars with abnormal surface compositions may not be quite those found using scaled solar composition models as the ultraviolet line blanketing and backwarming depend on the details of the composition in a very complicated manner. If the composition is metal-rich relative to solar, then the ultraviolet line blanketing and backwarming are greater than for a solar composition star and the star looks hotter in the optical region. However, if the abundances of the iron-peak elements are solar on average, but still have very non-solar values, it is difficult to predict what happens without detailed calculations as the line opacities of each species are somewhat different. The uncertainty in effective temperature due to this possibility increases that quoted above by a little.

Figure 1 is an HR diagram with the stellar evolutionary tracks from Schaller et al. (1992) for several stellar masses between 2 and $5 M_{\odot}$ and the locations of $\mathrm{HgMn}$ (x's) and Am stars (o's). These tracks were calculated for a solar metallicity $(Y=0.300$ and $Z=0.020)$ with an overshooting parameter $\alpha_{\text {over }}=0.20$. The stellar names and plotted values are given in Table 1 along with references to the optical region abundance analyses.

From this diagram we can conclude

1. Both HgMn and Am stars occur on the same evolutionary tracks in the mass range 2.5 to $2.9 M_{\odot}$. Mass loss does not significantly change the masses of these stars during their main sequence band lifetimes. Here $\mathrm{HgMn}$ stars are closer to the ZAMS and Am stars populate the same evolutionary tracks at a later time. Thus these coolest $\mathrm{HgMn}$ stars must evolve into the hottest Am stars. The errors in $\log L / L_{\odot}$ from the Hipparcos parallaxes for those stars on both sides of the boundary are insufficient to make a significant difference in the stellar positions to change this conclusion. A $2.5 M_{\odot}$ star such as $v \mathrm{Cnc}$ apparently spends a smaller fraction of its main sequence evolution as a $\mathrm{HgMn}$ star than does a $2.9 M_{\odot}$ star such as $28 \mathrm{Her}$ or HR 4487. The coolest HgMn stars also tend to be the most rare earth rich of their class and the Am stars have many members displaying a variety of rare earth element lines.

At classification dispersion, an Am star is an A or early F type star for which no unique spectral type can be defined. The strength of the Ca II K line does not correspond to those of the Balmer or metal lines (Roman et al. 1948). Bidelman later noted that in these stars at higher dispersion Sc II $\lambda 4246$ and $\lambda 4320$ were anomalously weak and offered an alternative characterization of such stars (Jaschek \& Jaschek 1987). Conti (1965) following this observation used the line ratio Sc II $\lambda 4246 / \mathrm{Sr}$ II $\lambda 4215$ to separate the Am stars as Sr II is enhanced and Sc II is quite weak in Am stars. As a result he extended the class to higher values of $T_{\text {eff }}$. Most Am stars which evolve from HgMn stars are the so called "hot" Am stars of Conti (1965). His prototypes included 68 Tau, 21 Lyn, $\phi$ Aql, o eg, $\beta \mathrm{UMa}, \omega \mathrm{UMa}$, and $\sigma$ Aqr, but not $\theta$ Leo which we list in Table 1 .

Abt (1961) discovered that all Am stars are spectroscopic binaries and most have periods less than 100 days. The few Am stars that are not known to be binaries can be considered to be seen pole-on. Our inference that the $\mathrm{HgMn}$ and the Am stars form a single class suggests at least the coolest HgMn stars might also belong to binary systems as class properties should smoothly change with physical parameters. However, Gerbaldi et al. (1985) concluded that the binary statistics for $\mathrm{HgMn}$ stars were essentially the same as for the corresponding normal main sequence stars. Still it might be useful to look at this question again as with high dispersion, high signal-to-noise spectroscopy, weak lines from additional companions to $\mathrm{HgMn}$ stars with large differences in the ratios of the strengths of the primary and secondary stars can be found (see, e.g., Ryabchikova et al. 1998 who found the companion to $\kappa \mathrm{Cnc})$.

Of all the fundamental stars only Sirius A, spectral type A1 Vm, has a location in Fig. 1 close to the HgMn - Am star boundary (Smalley \& Dworetsky 1995). It falls below the least massive HgMn star, $v$ Cnc, close to the ZAMS. This confirms that the lower bound for $\mathrm{HgMn}$ star masses is about 2.5 $\mathrm{M}_{\odot}$.

2. $33 \mathrm{Gem}$, which is often taken to be the hottest $\mathrm{HgMn}$ star, is the most luminous star of the sample, and 3 Cen $\mathrm{A}$, which is often considered to be a star related to the $\mathrm{HgMn}$ stars and is the hottest of the stars studied, have masses near $5 M_{\odot}$. Although all HgMn stars have Mn II lines stronger than expected for their spectra types, in practice this classification often depends on the presence or absence of $\mathrm{Hg}$ II 13984 . But $53 \mathrm{Tau}$ which has no such line is included in the class. As 3 Cen A also has a significant $\mathrm{Mn}$ overabundance it can be considered to be very hot class member. Thus the HgMn stars have masses between 2.5 and $5 M_{\odot}$ while the studied Am stars are between 2.0 and $2.9 M_{\odot}$. There may be Am stars with smaller masses since the sample in Fig. 1 is not complete for the coolest class members.

Another potential minor complication is that once $T_{\text {eff }}$ drops below about $9700 \mathrm{~K}, \mathrm{Fe}$ I $\lambda 3983.9564$ begins to appear in stellar spectra and can obscurate the question of the presence of $\mathrm{Hg}$ II $\lambda 3983.96$ (terrestrial). The isotopic shifts of the various components might provide some clues. There are also other possible weak blending components which can appear, but the Fe I line predominates and the equivalent width of this line is much smaller than those $\mathrm{Hg}$ II lines in the HgMn stars. In such cases one could look for the strongest $\mathrm{Hg}$ I line in the optical $\lambda 5460.742$ or the two weaker lines in the photographic of multiplet 1 . Unfortunately the analyses in Table 1 do not contain the relevant spectral region for $\lambda 5461$. 


\section{HgMn and Am Stars}

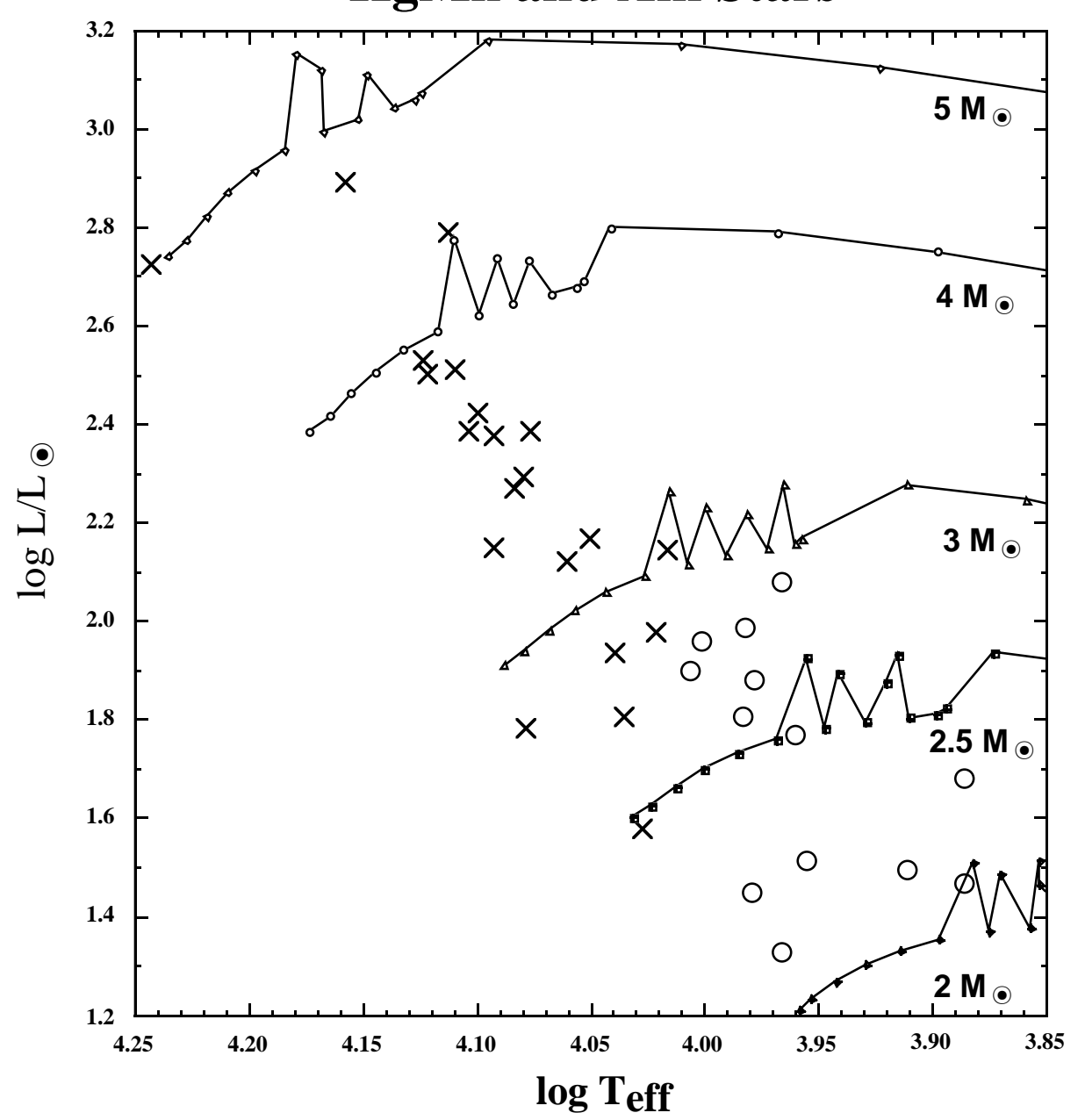

Fig. 1. The HR diagram showing the location of HgMn (x's) and Am stars (o's). The stellar evolutionary tracks from Schaller et al. (1992) were calculated for a solar metallicity $(Y=0.300$ and $Z=0.020)$ with an overshooting parameter $\alpha_{\text {over }}=0.20$. Each is labelled with its stellar mass.

3. Although a few stars are close to the ZAMS such as 3 Cen A, 53 Tau, and HR 7775, most are displaced to the right in Fig. 1 which means that they have begun to evolve away from the ZAMS. These three stars have some anomalous abundances relative to other class members. Thus it may take some time for most class members to become recognized as $\mathrm{HgMn}$ or Am stars. In this regard Woolf \& Lambert (1999) report the detection of three HgMn stars in the Orion OB1 Association which shows that such stars can be found 1.7 million years after they left ZAMS. The HgMn and Am stars are all located in the main sequence band which is a slow phase of nuclear burning.

\section{Statistical analyses}

When we looked at the abundances of stars whose positions in Fig. 1 place them close to one another, sometimes as for $\kappa$ Cnc and HR 7361 the agreement is usually within 0.2 dex, but more often it is not. This may be due to errors in the HR diagram positions, important hidden variables, undetected binarity, slight differences in initial conditions, chaos, and/or atmospheric fluxes effects due to non-solar compositions. If there is a hidden variable, it could be the time when the processes which produce the anomalous abundances begin to operate. If such processes produce observable effects only some time after the star is sufficiently slowly rotating and is sufficiently close to the ZAMS in its evolution, then it is likely to relate to time at which this occurs as most stars are thought to be born with a substantial amount of rotation. For stars in binary systems important factors could be the ratio of the mass components, their separation, their orbital parameters, and the amount of gas in the system. Single slowly rotating stars can be produced by the random distribution of angular momentum in the process of formation. For a single star, slow rotation can depend on the interactions with its stellar siblings as well as with interstellar gas clouds.

We compared the abundances of HgMn and Am stars with the same masses: HR 7775 with $\beta$ UMa and $\pi$ Dra, and 53 Tau, HR 4487, 28 Her, and HR 7018 with $\sigma$ Aqr, $\omega$ UMa, 21 Lyn, and o Peg. The first comparison tends to yield the most obvious class differences while for the second individual peculiarities especially for 53 Tau are obvious. For both sets, the 
Table 1. Am and HgMn stars.

\begin{tabular}{|c|c|c|c|c|}
\hline Name & HD & $\log T_{\text {eff }}$ & $\log L / L_{\odot}$ & Analysis Reference \\
\hline \multicolumn{5}{|l|}{ Am Stars } \\
\hline $15 \mathrm{Vul}$ & 189849 & 3.886 & $1.68 \pm 0.04$ & Adelman et al. (1997) \\
\hline $32 \mathrm{Aqr}$ & 209625 & 3.886 & $1.47 \pm 0.05$ & Adelman et al. (1997) \\
\hline $6 \mathrm{Lyr}$ & 173648 & 3.911 & $1.50 \pm 0.02$ & Adelman et al. (1999) \\
\hline $68 \mathrm{Tau}$ & 27962 & 3.955 & $1.52 \pm 0.03$ & Adelman (1994b) \\
\hline$\pi$ Dra & 182564 & 3.960 & $1.77 \pm 0.03$ & Adelman (1996) \\
\hline 60 Leo & 95608 & 3.966 & $1.33 \pm 0.03$ & Adelman et al. (1999) \\
\hline$\theta$ Leo & 97633 & 3.966 & $2.08 \pm 0.04$ & Adelman (1988b) \\
\hline 21 Lyn & 58142 & 3.978 & $1.88 \pm 0.05$ & Adelman (1994b) \\
\hline$\phi$ Aql & 188728 & 3.979 & $1.45 \pm 0.04$ & Caliskan \& Adelman (1997) \\
\hline o Peg & 214994 & 3.982 & $1.99 \pm 0.06$ & Adelman (1988b) \\
\hline$\beta \mathrm{UMa}$ & 95418 & 3.983 & $1.80 \pm 0.01$ & Adelman (1996) \\
\hline$\omega \mathrm{UMa}$ & 94334 & 4.001 & $1.96 \pm 0.05$ & Caliskan \& Adelman (1997) \\
\hline$\sigma$ Aqr & 213320 & 4.006 & $1.90 \pm 0.09$ & Adelman \& Albayrak (1998) \\
\hline \multicolumn{5}{|c|}{ HgMn Stars } \\
\hline$v \mathrm{Cnc}$ & 77350 & 4.016 & $2.14 \pm 0.12$ & Adelman (1989) \\
\hline HR 7018 & 172728 & 4.021 & $1.98 \pm 0.05$ & Adelman et al. (2001) \\
\hline HR 7775 & 193452 & 4.027 & $1.58 \pm 0.09$ & Adelman (1994a) \\
\hline $28 \mathrm{Her}$ & 149121 & 4.035 & $1.80 \pm 0.08$ & Adelman \& Pintado (2000) \\
\hline HR 4487 & 101189 & 4.039 & $1.94 \pm 0.05$ & Adelman \& Pintado (2000) \\
\hline$\iota \mathrm{CrB} \mathrm{p}$ & 143807 & 4.051 & $2.17 \pm 0.06$ & Adelman (1989) \\
\hline$\phi \mathrm{Her}$ & 145389 & 4.061 & $2.12 \pm 0.03$ & Adelman et al. (2001) \\
\hline$v$ Her & 144206 & 4.077 & $2.39 \pm 0.06$ & Adelman et al. (2001) \\
\hline $53 \mathrm{Tau}$ & 27295 & 4.079 & $1.78 \pm 0.06$ & Adelman (1987) \\
\hline 14 Hya & 75333 & 4.080 & $2.29 \pm 0.09$ & Adelman \& Pintado (2000) \\
\hline HR 7245 & 178065 & 4.084 & $2.27 \pm 0.18$ & Adelman \& Pintado (2000) \\
\hline$\mu$ Lep & 33904 & 4.093 & $2.38 \pm 0.04$ & Adelman \& Pintado (2000) \\
\hline HR 89 & 1909 & 4.093 & $2.15 \pm 0.11$ & Adelman et al. (1996) \\
\hline HR 4817 & 110073 & 4.100 & $2.42 \pm 0.08$ & Adelman \& Pintado (2000) \\
\hline$\pi^{1}$ Boo & 129174 & 4.104 & $2.39 \pm 0.08$ & Adelman (1992) \\
\hline HR 7664 & 190229 & 4.110 & $2.51 \pm 0.12$ & Adelman (1988a) \\
\hline HR 8349 & 207857 & 4.113 & $2.79 \pm 0.19$ & Adelman (1989) \\
\hline$\kappa \mathrm{Cnc}$ & 78316 & 4.122 & $2.50 \pm 0.12$ & Adelman \& Pintado (2000) \\
\hline HR 7361 & 182308 & 4.124 & $2.53 \pm 0.12$ & Adelman (1992) \\
\hline 33 Gem & 49606 & 4.158 & $2.89 \pm 0.21$ & Adelman et al. (1996) \\
\hline 3 Cen A & 120709 & 4.243 & $2.72 \pm 0.07$ & Adelman \& Pintado (2000) \\
\hline
\end{tabular}

Mn, Sr, Y, and Zr abundances are greater in the HgMn stars and $\mathrm{Hg}$ abundances are found only in the $\mathrm{HgMn}$ stars while microturbulences are greater for the Am stars.

\subsection{Graphical analysis}

In an attempt to overcome the individual stellar peculiarities, we examined how the values of various abundances and the microturbulence varied by adding these values for each star to the HR diagram. We concluded that:

$\mathrm{HgMn}$ stars have microturbulences between 0.0 and $1.2 \mathrm{~km} \mathrm{~s}^{-1}$ while the Am stars have values between 1.0 and $5.6 \mathrm{~km} \mathrm{~s}^{-1}$ with values rapidly becoming larger with decreasing temperature. In part this may mean that Am stars spend sufficient time in the main sequence band for their microturbulences to increase as their atmospheres slowly expand.
Often the He abundances are smallest for those stars nearest to the ZAMS. Although many Am stars have subsolar He, a few have solar values. The abundances from Mg II lines behave somewhat similarly. Those Am stars with abundances from S II lines have larger derived values than most HgMn stars. Most, but not all HgMn stars, have Sc abundances greater than solar while Am stars have values ranging from solar to much less than solar. Most $\mathrm{HgMn}$ stars are more $\mathrm{Ti}$ and $\mathrm{Cr}$ rich than a typical Am star which is Ti solar and Cr slightly rich.

The hotter HgMn stars are more Mn rich than the cooler $\mathrm{HgMn}$ stars which in turn are more Mn rich than the Am stars. Many $\mathrm{HgMn}$ and Am stars are marginally Fe rich with the coolest Am stars tending to be definely Fe rich. Most HgMn stars are Ni poor while most Am stars are Ni rich. The HgMn stars are Y rich to a greater extent than the Am stars.

$\mathrm{Hg}$ abundances of the $\mathrm{HgMn}$ stars are substantially overabundant as deduced from optical region studies, but those of 
Table 2. Significant correlations.

\begin{tabular}{|c|c|c|c|}
\hline Compared & Quanitities & $r$ & values \\
\hline $\log \mathrm{Mg} / \mathrm{H}$ & $\log \mathrm{T}_{\text {eff }}$ & -0.403 & 34 \\
\hline $\log \mathrm{Mg} / \mathrm{H}$ & $\xi$ & -0.405 & 34 \\
\hline $\log \mathrm{Mn} / \mathrm{H}$ & $\log T_{\text {eff }}$ & 0.802 & 34 \\
\hline $\log \mathrm{Mn} / \mathrm{H}$ & $\xi$ & -0.633 & 34 \\
\hline $\log \mathrm{Fe} / \mathrm{H}$ & $\log g$ & 0.394 & 34 \\
\hline $\log \mathrm{Fe} / \mathrm{H}$ & $\xi$ & 0.382 & 34 \\
\hline $\log g$ & $\xi$ & 0.370 & 34 \\
\hline $\log T_{\text {eff }}$ & $\xi$ & -0.675 & 34 \\
\hline $\log \mathrm{Mg} / \mathrm{H}$ & $\log \mathrm{He} / \mathrm{H}$ & 0.530 & 30 \\
\hline $\log \mathrm{Cr} / \mathrm{H}$ & $\log \mathrm{He} / \mathrm{H}$ & 0.387 & 30 \\
\hline $\log \mathrm{Mn} / \mathrm{H}$ & $\log \mathrm{He} / \mathrm{H}$ & -0.520 & 30 \\
\hline $\log T_{\text {eff }}$ & $\log \mathrm{He} / \mathrm{H}$ & -0.588 & 30 \\
\hline$\xi$ & $\log \mathrm{He} / \mathrm{H}$ & 0.444 & 30 \\
\hline $\log T_{\text {eff }}$ & $\log \mathrm{Si} / \mathrm{H}$ & 0.614 & 31 \\
\hline $\log \mathrm{Mn} / \mathrm{H}$ & $\log \mathrm{Ca} / \mathrm{H}$ & 0.431 & 27 \\
\hline$\xi$ & $\log \mathrm{Ca} / \mathrm{H}$ & -0.411 & 27 \\
\hline $\log \mathrm{Mn} / \mathrm{H}$ & $\log \mathrm{Sc} / \mathrm{H}$ & 0.703 & 29 \\
\hline $\log T_{\text {eff }}$ & $\log \mathrm{Sc} / \mathrm{H}$ & 0.674 & 29 \\
\hline$\xi$ & $\log \mathrm{Sc} / \mathrm{H}$ & -0.574 & 29 \\
\hline $\log \mathrm{Ti} / \mathrm{H}$ & $\log \mathrm{Mn} / \mathrm{H}$ & 0.561 & 33 \\
\hline $\log \mathrm{Ti} / \mathrm{H}$ & $\log T_{\text {eff }}$ & 0.616 & 33 \\
\hline $\log \mathrm{Ti} / \mathrm{H}$ & $\log \mathrm{Y} / \mathrm{H}$ & 0.487 & 32 \\
\hline $\log \mathrm{Mn} / \mathrm{H}$ & $\log \mathrm{Ni} / \mathrm{H}$ & -0.734 & 28 \\
\hline $\log \mathrm{Fe} / \mathrm{H}$ & $\log \mathrm{Ni} / \mathrm{H}$ & 0.648 & 28 \\
\hline $\log \mathrm{Ni} / \mathrm{H}$ & $\log T_{\text {eff }}$ & -0.597 & 28 \\
\hline$\xi$ & $\log \mathrm{Ni} / \mathrm{H}$ & 0.727 & 28 \\
\hline $\log \mathrm{Cr} / \mathrm{H}$ & $\log \mathrm{Sr} / \mathrm{H}$ & 0.409 & 29 \\
\hline $\log g$ & $\log \mathrm{Sr} / \mathrm{H}$ & 0.506 & 29 \\
\hline $\log \mathrm{Cr} / \mathrm{H}$ & $\log \mathrm{Y} / \mathrm{H}$ & 0.429 & 32 \\
\hline $\log \mathrm{Mn} / \mathrm{H}$ & $\log \mathrm{Y} / \mathrm{H}$ & 0.739 & 32 \\
\hline $\log T_{\text {eff }}$ & $\log \mathrm{Y} / \mathrm{H}$ & 0.655 & 32 \\
\hline $\log \mathrm{Y} / \mathrm{H}$ & $\xi$ & -0.644 & 32 \\
\hline $\log \mathrm{Ti} / \mathrm{H}$ & $\log \mathrm{Zr} / \mathrm{H}$ & 0.533 & 25 \\
\hline $\log \mathrm{Mn} / \mathrm{H}$ & $\log \mathrm{Zr} / \mathrm{H}$ & 0.793 & 25 \\
\hline $\log T_{\text {eff }}$ & $\log \mathrm{Zr} / \mathrm{H}$ & 0.666 & 25 \\
\hline$\xi$ & $\log \mathrm{Zr} / \mathrm{H}$ & -0.416 & 25 \\
\hline
\end{tabular}

some Am stars as found from ultraviolet lines while still $\mathrm{Hg}$ overabundant are considerably less so (Wahlgren \& Dolk 1998). The boundary between the these two types of stars in the HR diagram (in Fig. 1) is a hence a major test of the theories which try to explain the anomalous abundances of such stars.

\subsection{Linear correlation analysis}

Our interlocking of the HgMn and Am stars means that it is now justified to perform a linear correlation analysis using the effective temperatures, surface gravity, microturbulences, and abundances for all the stars of Table 1. This analysis is also of value in guiding linear regression analyses. Table 2 presents the positive results of the significant correlations for the optical abundances found in most objects. A correlation is regarded as significant if there is less than one chance in 20 for it to be due to chance (Bevington \& Robinson 1992) which means for 34 values, $r=0.339$, for 32 values, $r=0.349$, for 30 values, $r=0.361$, for 28 values, $r=0.380$, and for 25 values, $r=$ 0.395 .

First, the abundances from Mg II, Cr II, Mn II, and Fe II lines, the effective temperature $\left(T_{\text {eff }}\right)$, surface gravity $(\log g)$, and microturbulence $(\xi)$ were correlated against one another. Then the values of these quantitites were also correlated against the abundances from He I, Si II, Ti II, Ca II, Sc II, Ni II, Sr II, Y II, and Zr II lines. In Table 2, certain quantities are involved in many correlations, the most common quantities are microturbulence 11 times, effective temperature 10 times, and $\log \mathrm{Mn} / \mathrm{H}$ 8 times.

\subsection{Linear regression analysis}

Regression analysis (see, e.g., Neter et al. 1996) is a method of examining the relationship between one or more predictor variables and an outcome variable. Mathematically, it can be thought of as being given an $n$-dimensional scatter-plot of the data and drawing a line through it such that the line comes as close as possible (according to some criteria) to all the points. The line that one draws characterizes the outcome or dependent variable as a function of the predictor or independent variables. With this line is associated a $P$-value, which measures the statistical significance of the regression, i.e., whether a relationship has been demonstrated to be likely to exist. $P$-values range between 0 and 1 . The closer the $P$-value is to 0 , the more confident we are that the relationship actually exists. $P$-values greater than some value $\alpha$ (usually, but not necessarily, taken to be 0.05 ) indicates that the relationship has not been demonstrated to exist. How well the line fits the data is measured by the adjusted coefficient of multiple determination $\left(R_{\text {adj }}^{2}\right)$, which can have any value from less than 0 to $1 . R_{\text {adj }}^{2}$ can be interpreted as the fraction of variance accounted for by the model. The closer the value of $R_{\text {adj }}^{2}$ is to 1 , the better the fit of the regression line. An $R_{\text {adj }}^{2}$ smaller than 0 indicates an extraordinarily bad fit. In principle, the formula for the regression line can be anything one wishes, but as all possible models cannot be examined, a small, well-defined set is typically searched. In addition to overall tests of model fit, regression analysis also allows one to calculate $P$-values for any parameter. Any $P$-value greater than $\alpha$ is generally considered to indicate that the variable in question is not contributing significantly to the model.

We used SAS 6.12 for Macintosh to search for the best models using linear combinations of $\log T_{\text {eff }}, \log L / L_{\odot}$, microturbulence $(\xi)$, and (because of some suggestive scatter-plots) the square of microturbulence as predictor variables and log elemental abundances as outcome variables. An $\alpha$ of 0.05 was used. Table 3 shows the best models calculated for each abundance tested. The quality of the models improves as one proceeds down the table. Some predictor variables which make only small improvements in the fit may be used in the best 
Table 3. Best Linear regression models.

\begin{tabular}{lcrrrrrrrrrrr}
\hline \hline Dependent & $P$ & $R_{\text {adj }}^{2}$ & inter- & coefficient & $P$ & coefficient & $P$ & coefficient & $P$ & coefficient & $P$ \\
Variable & model & & cept & $\log T_{\text {eff }}$ & $\log T_{\text {eff }}$ & $\log L / L_{\odot}$ & $\log L / L_{\odot}$ & $\xi$ & $\xi$ & $\xi^{2}$ & $\xi^{2}$ \\
\hline $\log \mathrm{Si} / \mathrm{H}$ & 0.7344 & -0.0687 & -5.993 & 0.3457 & 0.7515 & 0.0182 & 0.9203 & 0.1173 & 0.1856 & -0.0221 & 0.2035 \\
$\log \mathrm{Cr} / \mathrm{H}$ & 0.1110 & 0.0763 & -16.295 & 2.8122 & 0.0396 & -0.5052 & 0.0536 & & & & \\
$\log \mathrm{Fe} / \mathrm{H}$ & 0.0614 & 0.0772 & -4.591 & & & & & 0.3281 & 0.0614 & \\
$\log \mathrm{Sr} / \mathrm{H}$ & 0.0431 & 0.1865 & 25.907 & -8.1985 & 0.0214 & & & -0.8581 & 0.0081 & 0.1081 & 0.0503 \\
$\log \mathrm{Mg} / \mathrm{H}$ & 0.0209 & 0.1295 & 2.387 & -1.7478 & 0.0209 & & & & & & & \\
$\log \mathrm{Ca} / \mathrm{H}$ & 0.0033 & 0.2426 & -5.369 & & & & & -0.1544 & 0.0033 & & \\
$\log \mathrm{Zr} / \mathrm{H}$ & 0.0005 & 0.3881 & -34.479 & 6.5871 & 0.0005 & & & & & & & \\
$\log \mathrm{He} / \mathrm{H}$ & 0.0003 & 0.3619 & 10.953 & -3.0868 & 0.0003 & & & & & & \\
$\log \mathrm{Ti} / \mathrm{H}$ & 0.0001 & 0.4196 & -31.713 & 6.5174 & 0.0003 & -0.5552 & 0.0598 & & & & \\
$\log \mathrm{S} / \mathrm{H}$ & 0.0001 & 0.6219 & 8.050 & -2.9912 & 0.1476 & -0.3955 & 0.2673 & 0.2402 & 0.0164 & & \\
$\log \mathrm{Sc} / \mathrm{H}$ & 0.0001 & 0.6459 & 5.525 & -4.4987 & 0.2330 & 1.9913 & 0.0003 & -0.5250 & 0.0508 & 0.0828 & 0.0650 \\
$\log \mathrm{Y} / \mathrm{H}$ & 0.0001 & 0.6495 & -7.095 & & & & & -1.3179 & 0.0001 & 0.2009 & 0.0001 \\
$\log \mathrm{Ni} / \mathrm{H}$ & 0.0001 & 0.6797 & 0.600 & -1.7388 & 0.2412 & & & 0.8204 & 0.0001 & -0.1205 & 0.0010 \\
$\log \mathrm{Mn} / \mathrm{H}$ & 0.0001 & 0.7856 & -27.246 & 5.1603 & 0.0097 & 0.6472 & 0.0458 & -0.5613 & 0.0007 & 0.1180 & 0.0003 \\
\hline
\end{tabular}

models. The fits are given in the form of the dependent variable equals the intercept plus the sum of coefficients times the important independent variables.

For the elemental abundances $\log \mathrm{Si} / \mathrm{H}, \log \mathrm{Cr} / \mathrm{H}$, and $\log \mathrm{Fe} / \mathrm{H}$, the $P$-value for the regression was greater than $\alpha$. In the case of $\log \mathrm{Si} / \mathrm{H}, R_{\text {adj }}^{2}$ for every model tested was less than 0 , indicating an utter lack of any demonstrable relationship to any of the predictor variables. In the case of $\log \mathrm{Cr} / \mathrm{H}$ and $\log \mathrm{Fe} / \mathrm{H}$, individual parameters are significant or close to being significant; in these cases the lack of overall significance may simply be due to there not being enough data in our sample to demonstrate a relationship between the predictor and outcome variables. No linear correlations with $\log \mathrm{S} / \mathrm{H}$ were presented in Table 2.

For the other elemental abundances except for $\log \mathrm{Sr} / \mathrm{H}$, the results of Table 2 serve as useful as predictors of Table 3 results. The significant linear correlations for the abundances with $\log T_{\text {eff }}$ and $\xi$ are mirrored. Often the best models which show a microturbulence dependence also have the square of the microturbulence dependence. The underlying phenomenon appears to have a primary $T_{\text {eff }}$ dependence with lesser dependence on the microturbulence (and its square) and somewhat less on the luminosity. The luminosity which is not correlated with other quantities in Table 2 , being a combination of $T_{\text {eff }}$ and $\log g$, plays a significant role for only a few elements.

\section{Conclusions}

That 2.5 to $2.9 M_{\odot} \mathrm{HgMn}$ stars evolve into Am stars requires a theoretical explanation. It clearly demonstrates that in their atmospheres the abundance of $\mathrm{Hg}$ must change. This effect is definitely mass dependent. The statistical analyses indicate that some correlations exist which implies that some general physical phenomena are at work, but they have yet to be fully characterized.
Acknowledgements. This work was supported in part by a grant from The Citadel Foundation to SJA. We thank Barry E. Adelman and David S. Leckrone for helpful conversations and useful suggestions as well as the referee Michael M. Dworetsky. This research has made use of the SIMBAD database operated at CDS, Strasbourg, France.

\section{References}

Abt, H. A. 1961, ApJS, 6, 37

Adelman, S. J. 1987, MNRAS, 228, 573

Adelman, S. J. 1988a, MNRAS, 235, 763

Adelman, S. J. 1988b, MNRAS, 230, 671

Adelman, S. J. 1989, MNRAS, 239, 487

Adelman, S. J. 1992, MNRAS, 258, 167

Adelman, S. J. 1994a, MNRAS, 266, 97

Adelman, S. J. 1994b, MNRAS, 271, 355

Adelman, S. J. 1996, MNRAS, 280, 130

Adelman, S. J., \& Albayrak, B. 1998, MNRAS, 300, 359

Adelman, S. J., \& Pintado, O. I. 2000, A\&A, 354, 899

Adelman, S. J., \& Rayle, K. E. 2000, A\&A, 355, 308

Adelman, S. J., Gulliver, A. F., \& Rayle, R. E. 2001, A\&A, 367, 597

Adelman, S. J., Philip, A. G. D., \& Adelman, C. J. 1996, MNRAS, 953

Adelman, S. J., Caliskan, H., Kocer, D., \& Bolcal, C. 1997, MNRAS, 288,470

Adelman, S. J., Caliskan, H., Cay, T., Kocer, D., \& Tektunali, G. H. 1999, MNRAS, 305, 591

Adelman, S. J., Young, J. M., \& Baldwin, H. E. 1984, MNRAS, 206, 649

Bessell, M. S., Castelli, F., \& Plex, B. 1998, A\&A, 333, 231

Bevington, P. R., \& Robinson, K. 1992, Data Reduction and Error Analysis for the Physical Sciences, 2nd. ed. (McGraw-Hill, New York)

Caliskan, H., \& Adelman, S. J. 1997, MNRAS, 288, 501

Conti, P. S. 1965, ApJ, 142, 1594

ESA 1997, The Hipparcos and Tycho Catalogues, SP-1200

Gerbaldi, M., Floquet, M., \& Hauck, B. 1985, A\&A, 146, 341

Hui-Bon-Hoa, A. 2000, A\&AS, 144, 203 
Jaschek, C., \& Jaschek, M. 1987, The Classification of Stars (Cambridge University Press, Cambridge)

Lemke, M. 1989, A\&A, 225, 125

Michaud, G. J., \& Proffitt, C. R. 1993, in Peculiar versus Normal Phenomena in A-type and Related Stars, ed. M. M. Dworetsky, F. Castelli, \& R. Faraggiana (San Francisco, ASP), ASP Conf. Ser., 44, 439

Napiwotzki, R., Schönberner, D., \& Wenske, V. 1993, A\&A, 268, 653 Neter, J., Wasserman, W., Kuter, M. H., \& Nachtsheim, C. 1966, Applied Linear Statistical Models (WCB McGraw-Hill, Boston)

Pintado, O. I., \& Adelman, S. J. 2001, X Latinaoamerican Astronomical Regional Meet., Cordoba, Argentina
Roman, N., Morgan, W. W., \& Eggen, O. 1948, ApJ, 107, 107

Ryabchikova, R., Kotchoukhov, O., Galazutdinov, G. F., \& Adelman, S. J. 1998, Contrib. Astron. Obs. Skalanta Pleso, 27, 258

Schaller, G., Schaerer, D., Meynet, G., \& Maeder, A. 1992, A\&AS, 96, 269

Smalley, B., \& Dworetsky, M. M. 1995, A\&A, 293, 446

Smith, K. C., \& Dworetsky, M. M. 1993, in Peculiar versus Normal Phenomena in A-type and Related Stars, ed. M. M. Dworetsky, F. Castelli, \& R. Faraggiana (San Francisco, ASP), ASP Conf. Ser., 44, 131

Wahlgren, G. M., \& Dolk, L. 1998, CoSka, 27, 314

Woolf, V. M., \& Lambert, D. L. 1999, ApJ, 520, L55 